\title{
A Study on the Local Electrochemical Processes Inside Cavities
}

\author{
X. T. SUN ${ }^{1,2, *}$, C. Y. ZHANG ${ }^{2}$ and X. S. CHEN $^{2}$ \\ ${ }^{1}$ Center for Precision Engineering, Shenzhen Institutes of Advanced Technology \\ Chinese Academy of Sciences, Shenzhen 518055, China \\ ${ }^{2}$ Center for Precision Engineering, Guangzhou Institutes of Advanced Technology \\ Chinese Academy of Sciences, Shenzhen 511458, China
}

${ }^{*}$ Corresponding author

Keywords: Potential variation, Cavity, Polarization.

\begin{abstract}
In this paper, a set of experiments scanning real potential variations inside cavities under current control were employed to characterize local electrochemical information. The limiting diffusion current and the critical currents sustaining the supersaturation of dissolved gas and completed oxide film-formation were selected as the reference currents. It is demonstrated that for gas-evolving electrode, only the bubble sealing the cavity remarkably increases the total potential variation within cavities. Combined with the theoretical analysis and measurement results, kinetic parameters range inside cavities can be indirectly derived.
\end{abstract}

\section{Introduction}

Cathodic polarization of a metal surface with a cavity or pit, as often happens under conditions of cathodic protection, electroplating and electrocoagulation technology $[1,2]$. The presence of cavities strongly accentuates the spatial non-uniformity of the potential and current distributions on the metal surface and within the depth of the cavity. Related modeling and simulation were based on theoretical idealizations of the actual physic-chemical conditions, so that the quantitative and predictive values of the local electrochemical information inside cavities were not emphasized $[3,4]$.

Fundamentally, the electrochemical information distribution inside cavities can be measured by scanning methods. However, the small area techniques that allow polarizing the microscopic surface areas do not apply to the scanning experiments $[5,6]$. In this work, we present a novel set of experiments scanning potential distributions inside cavies using a micro-reference electrode in typical three-electrode cell. Titanium is being studied since the experiences in its mechanisms of cathodic polarizations can help to design experiments. Our study has shown that the proposed approach is valuable for indirect derivation of the local electrode information but also for the direct evaluation of gas evolution and completed oxide film-formation effects on the electrochemical process evolutions within cavities in the practical conditions.

\section{Experiments}

Commercial titanium Grade 2 rods of $\varphi 7 \mathrm{~mm} \times 12 \mathrm{~mm}$ were used as the specimens. The investigated cavity with a diameter $2 \mathrm{~mm}$ and a depth $10 \mathrm{~mm}$ was drilled in the middle surface of cylindrical specimens. The solutions of $1 \%$ and $5 \%$ aqueous $\mathrm{NaCl}$ were prepared from analytical reagent grade chemicals and distilled water. 
The typical three-electrode cell was used. A Unisense micro-reference electrode REF-10 (silver/silver-chloride) with the outside tip diameter $8-12 \mu \mathrm{m}$ was fixed in the holder of MM33 micromanipulator mounted on a heavy laboratory stand. A platinum counter-electrode was processed into L-shape with a round cavity in the middle to allow the microcapillary pass through.

All scanning measurements were performed in constant current mode using a CHI660D potentiostat. The typical current densities and solution concentrations were chosen according to the results of potentiodynamic curves carrying out on the flat surface of specimens. Each potential-time monitoring experiment was repeated at least three times, with a fresh specimen being used for each test.

\section{Results and Discussion}
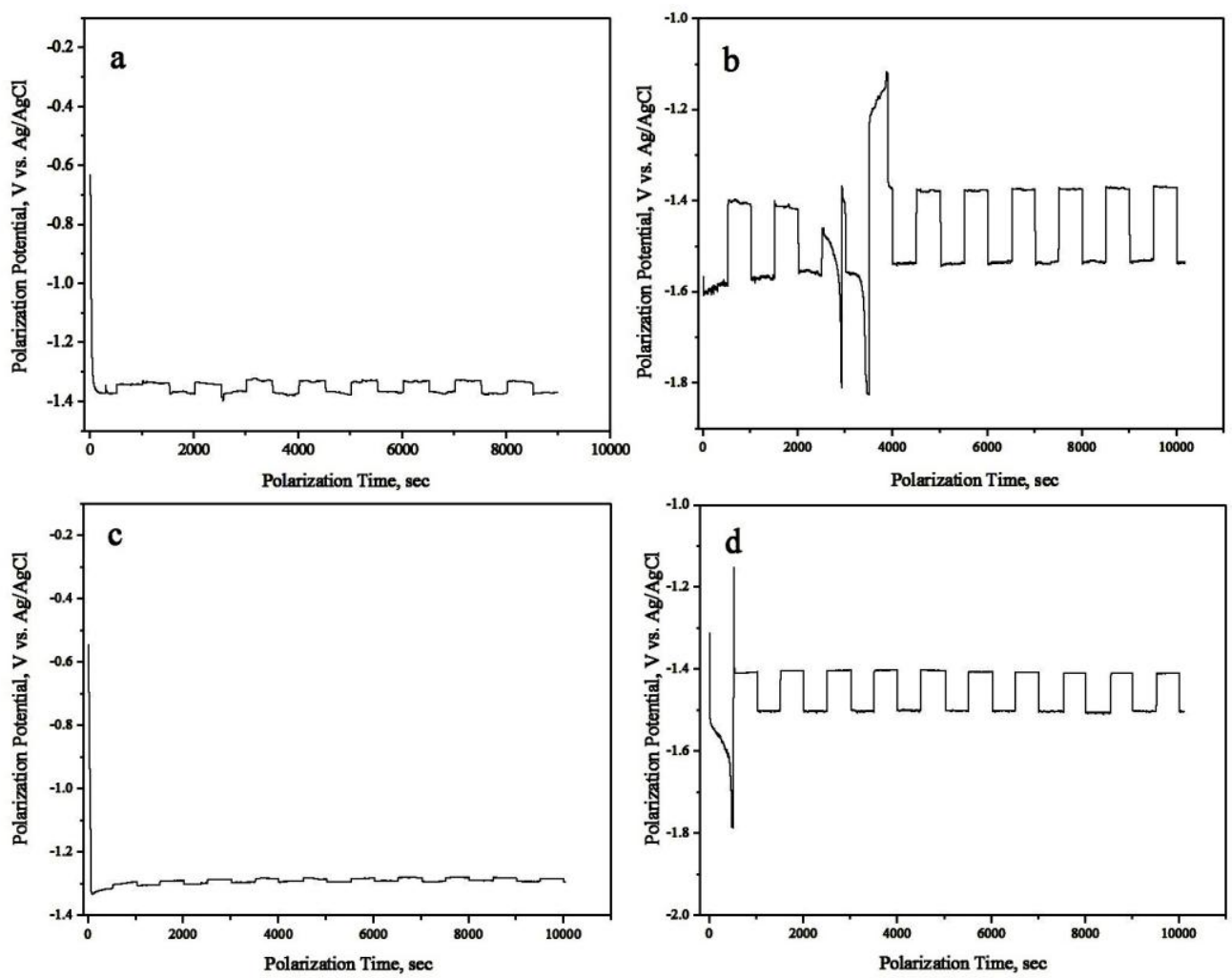

Fig. 1 Cathodic potential variations inside the 10mm-depth cavity in titanium electrode measured in $1 \%$ (a, b) and $5 \%(\mathrm{c}, \mathrm{d}) \mathrm{NaCl}$ at current densities of $10^{-4}(\mathrm{a}, \mathrm{c})$ and $10^{-3}(\mathrm{~b}, \mathrm{~d}) \mathrm{A} / \mathrm{cm}^{2}$.

Fig. 1 shows the variation amplitudes of the cathodic polarization potential within the $10 \mathrm{~mm}$-depth cavity of pure titanium specimens in $1 \%$ and $5 \% \mathrm{NaCl}$ at current densities of $10^{-4}$ and $10^{-3} \mathrm{~A} / \mathrm{cm}^{2}$. It is found that the curves show the regular "square wave" feature. In $1 \% \mathrm{NaCl}$ the variation amplitudes are about 50 and $200 \mathrm{mV}$ at $10^{-4}$ and $10^{-3}$ $\mathrm{A} / \mathrm{cm}^{2}$ respectively. The absolute potential at cavity opening is expectedly higher than that at cavity bottom. Anomalous potential variations at $10^{-3} \mathrm{~A} / \mathrm{cm}^{2}$ in the time range 2500 to 6000 seconds reach about $800 \mathrm{mV}$ as a result of hydrogen accumulation inside the cavity. During this time range the bubble coalescence, growth and detachment can be observed at the cavity opening. After the bubble departure, the potential quickly continue the previous value. It is important to note that for most of the cathodic polarization time bubbles with much smaller diameters $d_{b}$ than the cavity diameter $d_{c}$, continuously escape from the cavity. However, only when the bubbles coalesce to one 
larger bubble attaching and sealing the cavity opening, the total potential variation remarkably increases. In present experiment conditions, the net effect of the bubble evolution is the expected overpotential increase. The ohmic effects associated with electrode-surface screening by attached large bubbles are predominant. The large amount of small size bubbles have little effect on the total potential variation.

In $5 \% \mathrm{NaCl}$ the big potential variation about $700 \mathrm{mV}$ caused by hydrogen accumulation appears at the first 500 seconds of $10^{-3} \mathrm{~A} / \mathrm{cm}^{2}$. For the rest of monitoring time, the potential variation amplitudes are about $10 \mathrm{mV}$ and $100 \mathrm{mV}$ for $10^{-4}$ and $10^{-3}$ $\mathrm{A} / \mathrm{cm}^{2}$ respectively, lower than the values measured in $1 \% \mathrm{NaCl}$. The total overpotential variation $\Delta \eta_{(\mathrm{n})}$ between the cavity opening and the depth $h$ into the cavity can be expressed as Eq. (1):

$$
\Delta \eta_{(h)}=\Delta \eta_{Q \eta(h)}+\Delta \eta_{a(h)}+\Delta \eta_{c(h)}
$$

where the subscripts "Rl", "a" and "c" stand for "solution ohmic", "activation" and "concentration", respectively. With primary and secondary current distribution both considered, the activation overpotential variation $\Delta \eta_{a(h)}$ of hydrogen evolution can be given by the following equation:

$$
\Delta \eta_{a(h)}=b \log \frac{R_{(h)}+R_{p(h)}}{R_{(0)}+R_{p(o)}}
$$

where $b$ is Tafel constant of hydrogen evolution reaction, $R_{(0)}$ and $R_{(k)}$ are the resistances of the solution in the electrolytic cell and within the cavity, and $R_{p(0)}$ and $R_{p(h)}$ are the polarization resistances at the cavity opening and the depth $h$ into the cavity. The cathode polarization curves show the limiting diffusion current $l_{d}$ for oxygen is about $10^{-5} \mathrm{~A} / \mathrm{cm}^{2}$ in the present experiments. Therefore, at $10^{-4}$ and $10^{-3} \mathrm{~A} / \mathrm{cm}^{2}$ that are greater than ten times $I_{d}$ for oxygen, the presence of dissolved oxygen has little effect on hydrogen overpotential [7, 8]. Thus, the $\Delta \eta_{(\mathrm{k})}$ can be expressed by Eq. (3).

$$
\Delta \eta_{(\mathrm{h})}=\Delta \eta_{O l(\mathrm{~h})}+b \log \frac{R_{(h)}+R_{p(h)}}{R_{(0)}+R_{p(o)}}
$$

According to the resistivity diagram of $\mathrm{NaCl}$ solutions, the solution ohmic resistance $R_{0}$ and $R_{\hbar}$ are calculated as $12 \Omega$ and $10350 \Omega$ for $1 \% \mathrm{~g} / 1$ solution, and as $3 \Omega$ and $2866 \Omega$ for $5 \% \mathrm{~g} / \mathrm{l}$ solution, respectively. Besides, the Tafel constant $b$ is calculated as 0.134 by the following equation

$$
b=\frac{\phi_{2}-\phi_{1}}{\log i_{2}-\log i_{1}{ }^{x}}
$$

Based on these data, if only primary current distribution is considered, the $\Delta \eta_{a(h)}$ can be calculated as $400 \mathrm{mV}$, which is much higher than the experiment values without the presence of the larger bubble. Therefore, in the current range from $10^{-4} \mathrm{~A} / \mathrm{cm}^{2}$ to $10^{-3}$ $\mathrm{A} / \mathrm{cm}^{2}$, the secondary current distributions play a significant role. Consequently, with the primary and secondary current distribution both considered, the average current density $j_{\text {ave }}$ and the $R_{p(0)}$ values range can be indirectly derived. At $10^{-4} \mathrm{~A} / \mathrm{cm}^{2}$, the $j_{\text {ave }}$ is less than $7.7 \times 10^{-6} \mathrm{~A} \cdot \mathrm{cm}^{-2}$ and $3.1 \times 10^{-5} \mathrm{~A} \cdot \mathrm{cm}^{-2}$ for $1 \% \mathrm{~g} / \mathrm{l}$ and $5 \% \mathrm{~g} / \mathrm{l} \mathrm{NaCl}$ solution, respectively; the $R_{p(0)}$ is higher than $4385 \Omega$ and $335 \Omega$ for $1 \% \mathrm{~g} / 1$ and $5 \% \mathrm{~g} / \mathrm{l} \mathrm{NaCl}$ solution, respectively. Thus, the internal surface within the cavity has lower current densities by one or two orders of magnitude than the cavity opening surface. Obviously, the more testing depths applied in the experiment design, the more accurate values can be calculated. 


\section{Summary}

In summary, this paper shows a novel approach of scanning real potential variations for the visualization of local polarization processes inside cavities in titanium electrode. The experimental results validate those anomalous potential distributions associated with gas evolution and completed oxide film formation that remained unverified up to now. Combined with the theoretical analysis, these measurements allow indirect derivation of local electrode information by means of increasing the testing points of different depths within the cavity.

\section{Acknowledgement}

This research was supported by the Basic Research Foundation of Shenzhen City, China (No. JCYJ20150521094519494) and the Science and technology projects of Guangzhou City, China (No. 201604020147).

\section{References}

[1] M.H. Parsa, S.R. Allahkaram, A.H. Ghobadi, J. Pet. Sci. Eng. 72 (2010) 215.

[2] J.L. Nava, M.T. Oropeza, C. Ponce de León, J. González-García, A.J. Frías-Ferrer, Hydrometallurgy. 91 (2008) 98.

[3] L. Martens, K. Hertwig, Electrochim. Acta. 40(1995) 387.

[4] C.T.J. Low, E.P.L. Roberts, F.C. Walsh, Electrochim. Acta. 52 (2007) 3831.

[5] K.J. Aoki, C.F Zhang, J.Y Chen, T. Nishiumi, J. Electroanalytical Chem. 706 (2013) 40.

[6] T. Suter, H. Böhni, Electrochim. Acta. 47 (2001) 191.

[7] C.D Hall Jr, N. Hackerman, J. Phys. Chem. 57 (1953) 262.

[8] N. Hackerman, C.D Hall Jr, J. Electrochem. Soc. 101 (1954) 321. 